\title{
A cicatrização do manguito rotador
}

\section{Rotator Cuff Healing}

\author{
Ildeu Afonso de Almeida Filho ${ }^{1,2}$ Daniel Andrade Coelho ${ }^{2,3}$ \\ ${ }^{1}$ Departamento de Ortopedia, Hospital Felício Rocho, Belo Horizonte, \\ MG, Brasil \\ ${ }^{2}$ Hospital Vera Cruz, Belo Horizonte, MG, Brasil \\ ${ }^{3}$ Hospital Semper, Belo Horizonte, MG, Brasil \begin{abstract}
Department of Orthopaedics, Hospital Felício Rocho, Av. do Contorno, 9530, Barro Preto, Belo Horizonte, MG, 30190-130, Brasil
\end{abstract} \\ Endereço para correspondência Ildeu Afonso de Almeida Filho, \\ (e-mail: ildeu1968@gmail.com).
}

Rev Bras Ortop 2021;56(3):291-298.

\section{Resumo \\ Palavras-chave \\ - lesões do manguito rotador/diagnóstico \\ - lesões do manguito rotador/ epidemiologia \\ - lesões do manguito rotador/cirurgia \\ - cicatrização}

O presente artigo aborda de forma ampla os aspectos que interferem no processo de cicatrização do manguito rotador. São considerados hábitos de vida como tabagismo e alcoolismo, fatores sistêmicos como diabetes mellitus, hipertensão arterial e obesidade bem como fatores locais, dentre os quais aqueles relacionados ao pré, per e pós operatório. A partir de uma extensa revisão da literatura, com a citação de 60 artigos científicos tanto da literatura ocidental como oriental, os autores pretendem aprofundar no tema trazendo para a prática médica condutas embasadas em novos conceitos estabelecidos.

The present article broadly addresses the aspects that interfere with the healing process of the rotator cuff. Life habits, such as smoking and alcoholism, are considered, systemic factors such as diabetes mellitus, hypertension, and obesity, as well as local factors, among which are those related to the pre, peri, and postoperative periods. From an extensive literature review, with the citation of 60 scientific articles from both Western and Eastern literature, the authors intend to deepen the theme by bringing to medical practice conducts based on new established concepts.

\section{Introdução}

A incidência das lesões do manguito rotador tem crescido exponencialmente à medida que aumenta a expectativa de vida da população. Várias explicações técnicas têm sido dadas visando justificar esse aumento. A questão gira em torno dos aspectos degenerativos, relacionados ao processo de envelhecimento do organismo, associados a alguns hábitos de vida como a prática esportiva, profissão, a dieta, uso de medicamentos, e a presença de doenças associadas, além de questões genéticas, ainda pouco levadas em consideração nas publicações até o presente. As variações anatômicas recebido

11 de Fevereiro de 2020

aceito

15 de Abril de 2020

Publicado on-line

Setembro 24, 2020
DOI https://doi.org/

10.1055/s-0040-1713764. ISSN 0102-3616. (c) 2020. Sociedade Brasileira de Ortopedia e Traumatologia. All rights reserved.

This is an open access article published by Thieme under the terms of the Creative Commons Attribution-NonDerivative-NonCommercial-License, permitting copying and reproduction so long as the original work is given appropriate credit. Contents may not be used for commercial purposes, or adapted, remixed, transformed or built upon. (https://creativecommons.org/ licenses/by-nc-nd/4.0/)

Thieme Revinter Publicações Ltda., Rua do Matoso 170, Rio de Janeiro, RJ, CEP 20270-135, Brazil 


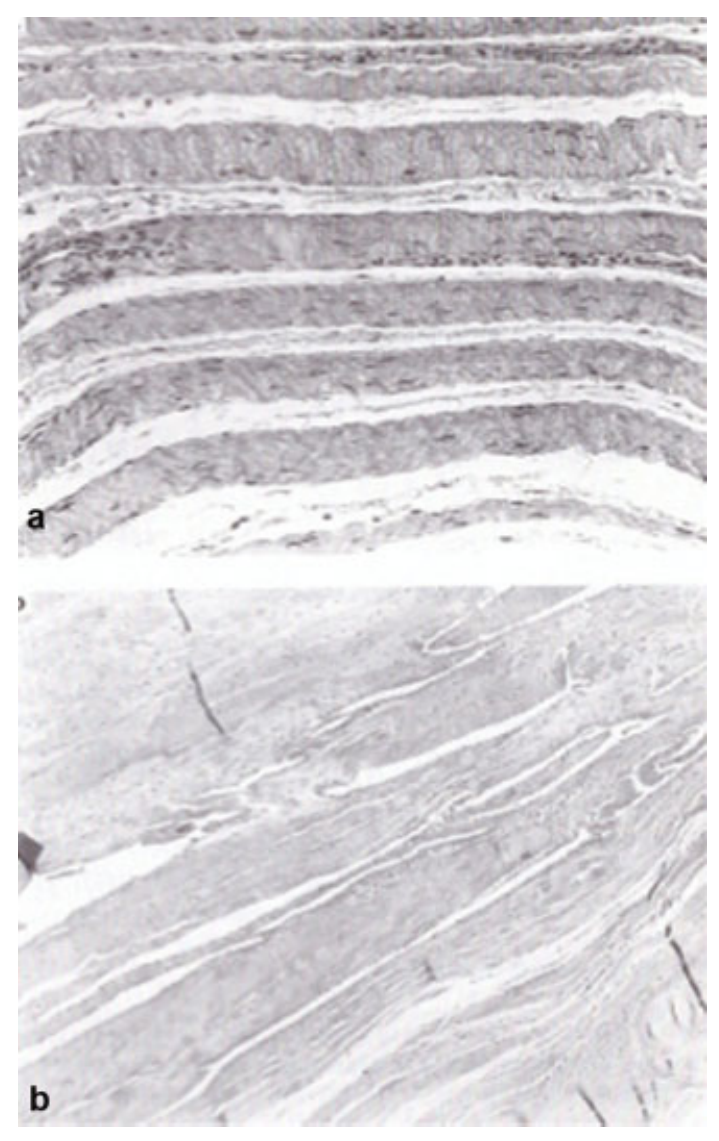

Fig. 1 No jovem, a inserção tendínea se organiza em camadas distintas (a) no idoso ocorre a sua desorganização (b).

também participam desse processo. Portanto, são muitos os fatores que podem vir a influenciar o desenvolvimento de lesões nos tendões do manguito rotador.

Hamada et al. ${ }^{1}$ estudaram a histologia do tendão supraespinal humano, subdividindo-o em porções e observaram que, no jovem, o tendão se organiza em camadas onduladas e que, no paciente mais velho, esse padrão se modifica para homogêneo, edemaciado e com degeneração hialina (- Figura 1).

Uthoff e Ishii ${ }^{2}$ analisaram a histologia da inserção tendinosa no tubérculo maior e observou, no jovem, a existência de um padrão de organização em camadas. No idoso, ocorre uma desorganização considerada como processo degenerativo (-Figura 2). O autor descreveu a reação inflamatória existente na lesão parcial do manguito rotador como uma tentativa de o próprio organismo cicatrizá-la. Porém, Yamaguchi et al. ${ }^{3}$ estudaram histologicamente a evolução natural dessas lesões em uma série de 58 casos e, em nenhum deles, observou-se a redução do tamanho da lesão. Esse fato mostra que a cicatrização espontânea do manguito rotador é algo que não deve ser esperado pelos pacientes e muito menos pelos cirurgiões.

O progressivo aumento na incidência de lesões na população em geral nos leva a perguntar sobre o que é considerado normal para uma determinada idade? Quantos portadores de lesão do manguito são assintomáticos? Milgron et al. ${ }^{4}$ afirmaram que a prevalência das lesões aumenta significativamente na população após os 70 anos de idade, sendo mais frequente no lado dominante, fato este comprovado por

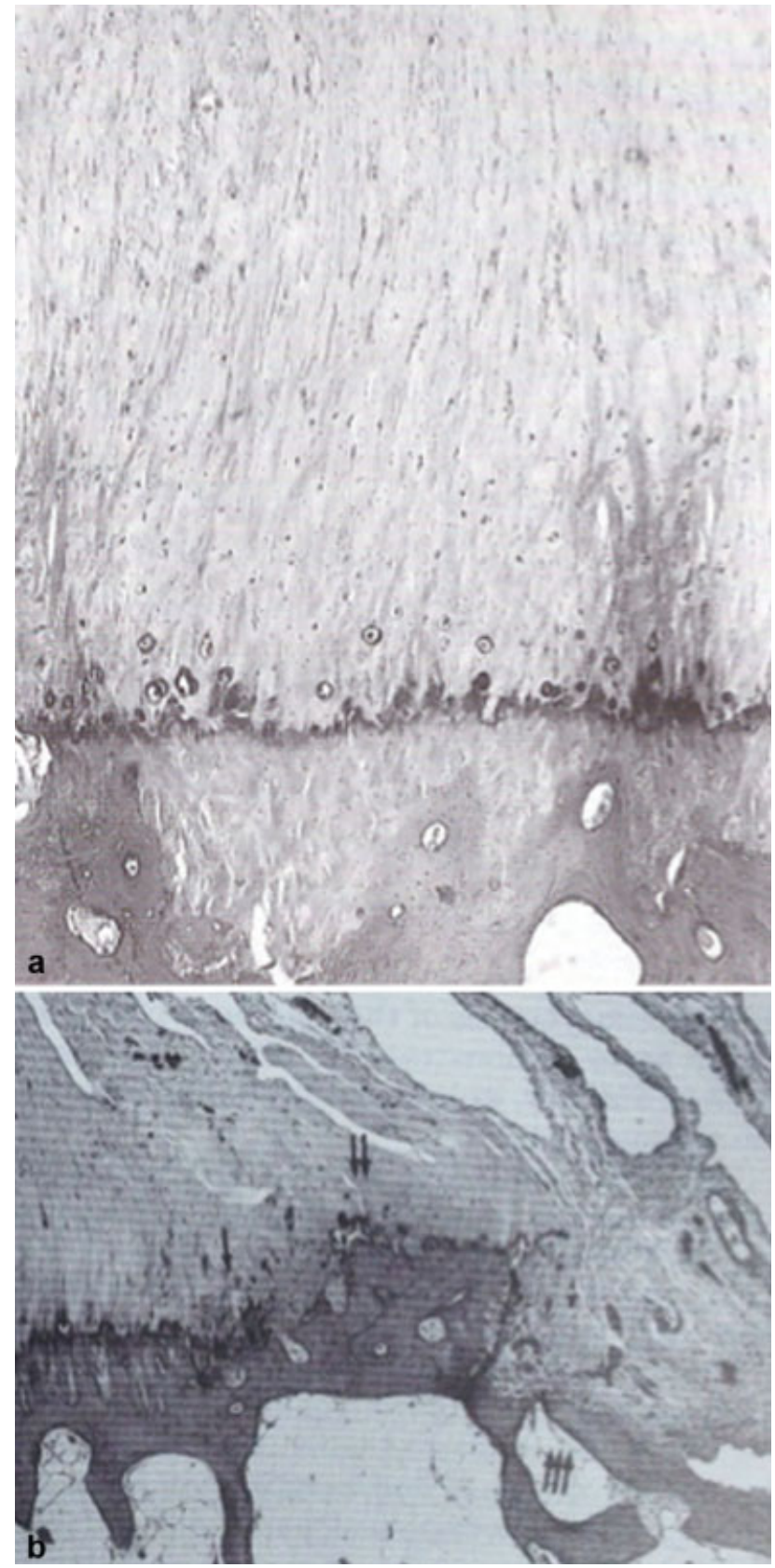

Fig. 2 Organização das fibras do tendão supraespinal em camadas no jovem, e a sua progressiva desorganização no adulto.

outros autores. ${ }^{5}$ Na sétima década de vida, essa incidência aumenta para mais de $80 \%$.

Durante as últimas duas décadas, os métodos de tratamento das lesões do manguito evoluíram substancialmente. As cirurgias, que antes eram feitas de forma aberta, passaram, na maioria dos centros de referência, a ser feitas de forma artroscópica. Diferentes tipos de suturas surgiram, e fios de alta resistência passaram a ser uma rotina.

\section{Fatores locais}

Os principais fatores associados à cicatrização do manguito rotador são o tamanho da lesão, qualidade muscular (atrofia 
e degeneração gordurosa), a qualidade do tendão, a presença de delaminação, e a morfologia do ombro.

\section{Tamanho da lesão}

A primeira característica avaliada por grande parte dos cirurgiões é o tamanho da lesão. A análise, contudo, deve ser realizada avaliando-se o tamanho da lesão no plano sagital e no plano coronal (retração medial ou encurtamento do tendão), uma vez que são características independentes com relação à taxa de cicatrização. Do ponto de vista geral, o aumento do tamanho da lesão influencia negativamente nas taxas de cicatrização, variando entre $97 \%$, para lesões pequenas, até apenas $6 \%$, para lesões grandes ou maciças, em algumas séries. ${ }^{6-10}$

\section{Tamanho avaliado no plano sagital}

Wylie et al., ${ }^{11}$ ao avaliar a taxa de cicatrização neste plano, mostraram que lesões $\geq 2,2 \mathrm{~cm}$ apresentavam índice de cicatrização de $49 \%$, e em lesões $\leq 2,2 \mathrm{~cm}$ a taxa subia para $71 \%$. Considerando este valor de corte, foi postulada a hipótese de que uma lesão que exceda $2 \mathrm{~cm}$ já apresenta envolvimento significante do infraespinal, ${ }^{12}$ o que altera a cinemática da cabeça umeral, gerando desbalanceamento no equilíbrio de forças. ${ }^{13}$ Esse fato colocaria o reparo em risco, justificando assim o risco aumentado de falha de cicatrização nas lesões maiores que $2,0 \mathrm{~cm}$.

\section{Tamanho avaliado no plano coronal (encurtamento)}

O encurtamento miotendinoso é considerado resultado direto da retração muscular. Contudo, Meyer et al. ${ }^{14}$ mostraram que apesar do encurtamento ser predominantemente da massa muscular, a retração é causada também por encurtamento do tendão em si, tanto devido à substância restante no tubérculo após a lesão inicial quanto, em estágios avançados, à reabsorção do próprio tendão. Em análise subsequente, Tashjian et al. ${ }^{6}$ mostraram que pacientes com lesões em que a junção miotendínea (JMT) está lateral a face da glenoide, a taxa de cicatrização foi de $93 \%$, enquanto nos pacientes com JMT pré-operatória medial à face da glenoide, a taxa de cicatrização foi de $55 \%$. A análise então concluiu que a posição da JMT é um fator independente na cicatrização. Do ponto de vista do tamanho da retração em si, Wylie et al. ${ }^{11}$ mostrou que lesões com encurtamento em relação ao footprint $\geq 2 \mathrm{~cm}$ apresentaram taxa de cicatrização de $47 \%$, em oposto a $76 \%$ de cicatrização nas lesões com retração $\leq 2 \mathrm{~cm}$.

\section{Qualidade muscular (atrofia e degeneração gordurosa)}

Quando se pensa em cicatrização do manguito rotador, um objetivo a ser alcançado é a cicatrização da interface tendãoosso. Dado esse fato, grande parte dos estudos foca em métodos para fortalecer ou interferir positivamente nessa interface. Entretanto, analisando lesões grandes, Jeong et al. ${ }^{15}$ observaram que a qualidade do músculo é mais importante do que a qualidade do tendão no índice de nova ruptura. De acordo com os autores, a taxa de ocupação do supraespinhal abaixo de $43 \%$ em relação à totalidade da fossa supraespinal e um grau de infiltração gordurosa $\geq 2$ segundo Goutallier são fatores de risco independentes para nova ruptura do manguito rotador, com sensibilidade de $98 \%$ e especificidade de 83,6\%. Uma revisão sistemática por Khair et al. ${ }^{16}$ corrobora este fato, indicando que a qualidade préoperatória do músculo tem papel importante na taxa de cicatrização do tendão. Estágios baixos de Goutallier (0-1) são associados a baixos índices de nova ruptura (25\%); em contrapartida, estágios altos (2-4) apresentam altos índices (59\%).

\section{Qualidade histológica e macroscópica do tendão}

Em relação à análise da qualidade histológica e macroscópica do tendão, foi sugerido que um tendão de qualidade pior acarretaria piores índices de cicatrização. Contudo, Mazzoca et al. ${ }^{17}$ mostraram que não há correlação entre a aparência macroscópica do tendão na artroscopia, ou na análise histológica, e a cicatrização. Mais ainda, mostraram que a aparência macroscópica não é correlacionada com a qualidade histológica do tendão, não havendo associação entre o grau de tendinopatia histológica, a aparência macroscópica e o índice de cicatrização ou resultados clínicos do reparo. Portanto, a aparência anormal não deve influenciar negativamente no esforço ou técnica do reparo.

\section{Delaminação}

Uma lesão completa crônica é considerada delaminada quando existe uma área de clivagem horizontal no tendão rompido. O manguito rotador é composto de cinco camadas, a saber: a mais superficial é composta por fibras do ligamento coracoumeral; a segunda camada é composta de fibras paralelas entre o supra e infraespinais; a terceira, por pequenos fascículos que fazem a interseção entre os tendões do manguito num ângulo de 45 graus; a quarta é composta por tecido conjuntivo frouxo extracapsular e que se conecta anteriormente com a porção profunda do ligamento coracoumeral; e, por último, a quinta camada é basicamente composta por cápsula articular. A segunda e terceira camadas, são mais espessas, apresentando diferentes direções das fibras de colágeno. ${ }^{18}$ É justamente entre estas camadas que ocorre a delaminação do tendão. Em um estudo recente, Boileau et al. ${ }^{19}$ mostraram uma prevalência de $32 \%$ de delaminação, sendo que todas envolviam um componente posterior da camada profunda. Esse estudo mostrou que, de modo geral, a delaminação tem um efeito deletério no índice de cicatrização, principalmente em lesões grandes. Kwon et al., ${ }^{20}$ em um estudo com 1.043 pacientes, confirmaram a taxa de cicatrização significativamente menor em tendões delaminados, se a análise for realizada utilizando-se uma única variável. Entretanto, ao se realizar análise multivariável, notou-se que a delaminação não é fator de risco independente para falha da cicatrização.

\section{Morfologia do ombro}

Heuberer et al. ${ }^{21}$ analisaram a morfologia do acrônimo e a correlacionaram com a chance do desenvolvimento de impacto subacromial, lesão, e artropatia do manguito rotador. Foram três as maneiras de se avaliar a morfologia acromial, a saber: o ângulo crítico do ombro (ACO), o índice acromial (IA) e o ângulo de inclinação lateral do acrômio 


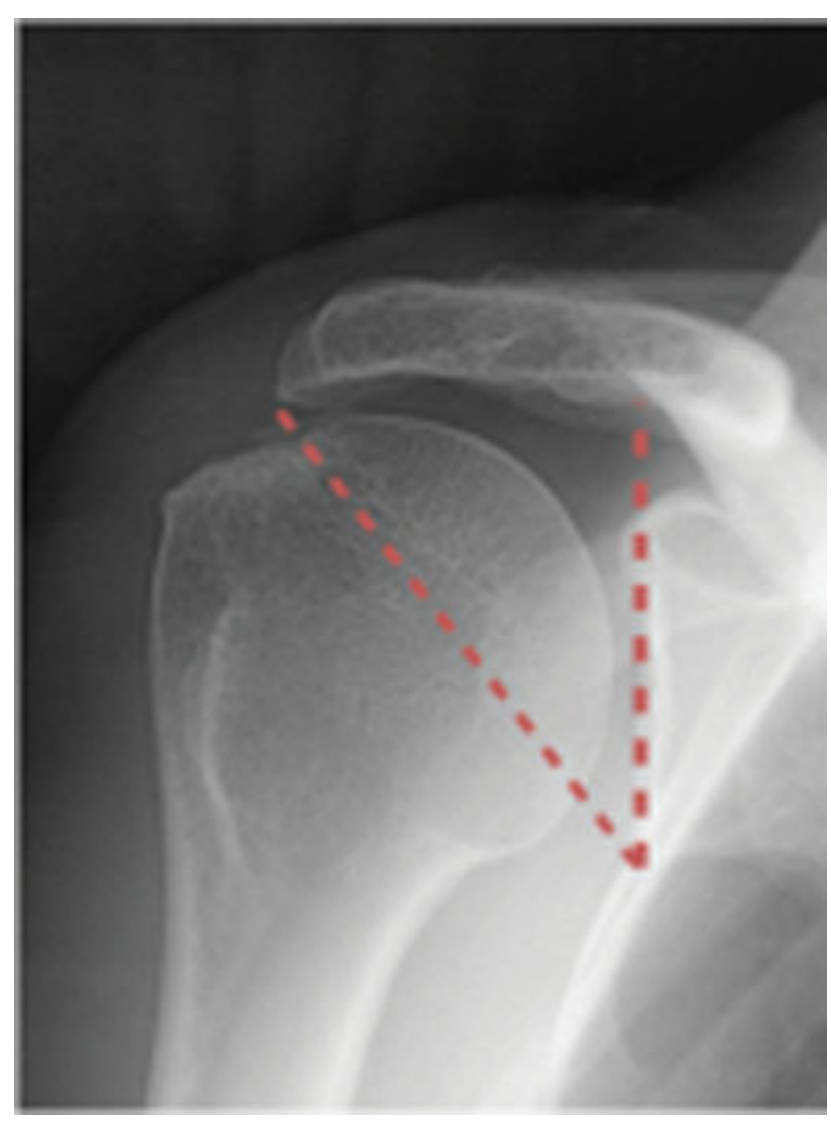

Fig. 3 Ângulo crítico do ombro.

(AILA). Os autores concluíram que a melhor maneira de se prever o aparecimento de lesões relacionadas ao manguito ou ao desenvolvimento de osteoartrose é através do ACO. Esse ângulo, descrito por Moor et al., ${ }^{22}$ apresenta grande benefício por combinar a extensão lateral do acrômio com a angulação da glenoide. É calculado na radiografia em anteroposterior, pelo ângulo entre a linha da borda inferior à borda superior da glenoide e a linha da borda inferior da glenoide ao ponto mais inferolateral do acrômio (-Figura 3). No estudo em questão, os autores demonstraram associação entre lesão do manguito rotador para $\operatorname{CSA}>35^{\circ}$ e artrose glenoumeral em $\operatorname{CSA}<30^{\circ}$. O fato foi demonstrado por outros autores. ${ }^{23}$

Um estudo biomecânico realizado por Gerber et al. ${ }^{24}$ se aprofundou no assunto, demonstrando que o CSA $>38^{\circ}$ requer um aumento de até $33 \%$ na atividade do supraespinhal para estabilização do ombro durante abdução de 6 a $61^{\circ}$. Esse fato poderia explicar os achados de Moor et al. ${ }^{22}$ e prever provável alteração nas taxas de cicatrização desses pacientes. De fato, Garcia et al. ${ }^{25}$ encontraram risco 14 vezes maior de nova ruptura em pacientes com $\operatorname{CSA}>38^{\circ}$. Uma revisão sistemática recente sobre o tema também confirmou a hipótese, mostrando que $\operatorname{CSA}>38^{\circ}$ é associado a menor taxa de cicatrização. ${ }^{26}$ Contudo, devido à heterogeneidade da literatura relevante na revisão em questão, a força deste achado é considerada limitada.

\section{Fatores sistêmicos}

Trataremos aqui dos diversos fatores sistêmicos que podem influenciar a cicatrização tendão-osso ou tendão-tendão.

O uso crônico do álcool é um importante fator no que se refere ao aumento da incidência de lesão bem como à gravidade da lesão do manguito rotador em ambos os sexos. A associação entre o consumo de álcool e as lesões do manguito rotador mostraram que, nos apreciadores de vinho, houve a presença de mais lesões maciças do que lesões pequenas. ${ }^{27}$

Já em relação ao tabagismo, é sabido que a nicotina atua na expressão da chamada MMP-9, que é uma enzima envolvida na degradação da matriz extracelular, em tenócitos. Esse fator leva à modificação do módulo de elasticidade do tendão sendo, segundo Park et al., ${ }^{28}$ um importante fator de risco para a propagação da lesão.

No que se refere à influência do diabetes mellitus no prognóstico do reparo do manguito, sabe-se que a persistência da hiperglicemia no pós-operatório aumenta a possibilidade de não cicatrização dos tendões reconstruídos. Portanto, o controle da glicemia após a cirurgia torna-se fundamental. ${ }^{29}$

A associação entre hipertensão arterial e lesões do manguito mostrou que, em relação aos normotensos, os hipertensos têm duas vezes mais lesões grandes e quatro vezes mais lesões maciças. ${ }^{30}$

O colesterol também causa influência no prognóstico ao aumentar a rigidez dos tendões por meio da modificação no seu módulo de elasticidade. ${ }^{31}$

Os pacientes obesos, aqueles com aumento do índice de massa corporal, também apresentam aumento da incidência e da gravidade das lesões do manguito rotador. ${ }^{32}$

Já os fatores imunobiológicos, ao interferir nos mecanismos associados à homeostase muscular, agravam o processo de degeneração gordurosa, o qual, por sua vez, determina na piora do prognóstico da cicatrização tendínea. ${ }^{33}$

Finalmente, a idade influencia negativamente o módulo de elasticidade do tendão.

Chung et al. ${ }^{34}$ analisaram os principais fatores prognósticos associados a maus resultados após a reconstrução cirúrgica do manguito rotador. A média de idade dos pacientes avaliados foi de 63,7 anos. Os autores observaram a incidência de $39,8 \%$ de falência no reparo das lesões do manguito rotador. Os piores fatores prognósticos considerados foram a degeneração gordurosa do músculo infraespinal e a redução do espaço subacromial pela migração cefálica da cabeça umeral.

Quadro 1 Maior cicatrização em pacientes mais jovens

\begin{tabular}{|l|l|l|l|}
\hline Grupos: & $<50$ & 51 a 60 & $>60$ anos \\
\hline N & 49 & 68 & 52 pacientes \\
\hline Cicatrização & $87,8 \%$ & $79,4 \%$ & $65,4 \%$ \\
\hline N-cicatrização & $12,2 \%$ & $20,6 \%$ & $34,6 \%$ \\
\hline
\end{tabular}


Cho et al. ${ }^{35}$ estudaram os fatores que afetam a integridade da reconstrução do manguito rotador. Dividiram os pacientes em três grupos, a saber: $<50$ anos de idade (grupo I), entre 51 e 60 anos (grupo II), e $>60$ anos de idade (grupo III). Observaram que, quanto maior a idade, menor é a incidência de cicatrização (Quadro 1). Apesar disso, o estudo recomenda a reconstrução das lesões em pacientes idosos em função da melhora clínica.

Veado et al. $^{36}$ avaliaram a integridade do reparo e a sua relação com a função nos ombros de pacientes submetidos à reconstrução artroscópica após os 65 anos de idade. Para o estudo, os autores utilizaram um mesmo ultrassonografista para a realização de todos os exames de imagem, e encontraram uma incidência de $75 \%$ de integridade tendínea com melhora do UCLA (do inglês, University of California Los Angeles Shoulder Score) de 17 pontos para 32 pontos o que representa $85 \%$ de resultados bons e excelentes. Houve também redução significativa do nível de dor quando utilizada a escala visual analógica.

Dezaly et al. ${ }^{37}$ realizaram trabalho prospectivo, comparativo, randomizado de dois grupos de pacientes. O grupo I foi submetido à reconstrução do manguito rotador, acromioplastia e tenotomia da porção longa do bíceps braquial. O grupo II foi submetido a tenotomia do bíceps e acromioplastia, sem reconstrução do manguito. Foram 103 paciente avaliados e, apesar do tempo de recuperação pós-operatória ter sido menor no grupo II, como era de se esperar, tanto o resultado funcional pelo escore de Constant (82 no grupo I e 73 no grupo II) quanto a distância subacromial medida nos exames de imagem foram favoráveis ao grupo I. Curiosamente, os melhores resultados foram observados nas reconstruções das lesões mais retraídas, sugerindo que pacientes idosos com lesões pequenas devam ser tratados conservadoramente.

Muitos estudos ${ }^{38}$ têm sugerido que o aumento da idade está relacionado a uma redução do potencial de cicatrização tendínea, enquanto estudos biomecânicos sugerem que a razão para isso deva estar em um ambiente desfavorável como a baixa perfusão tecidual e a redução do número de células indiferenciadas.

\section{Fatores pré-operatórios}

Os fatores sistêmicos e locais referidos anteriormente são inerentes ao paciente, e, portanto, de modo geral, de difícil controle por parte do cirurgião. Devido a isso, fatores relacionados às técnicas cirúrgicas se mostram de crucial importância nos resultados, já que nesse ponto o cirurgião pode atuar ativamente na melhora da cicatrização. Aqui, uma boa escolha, focada no conhecimento técnico, pode aumentar as taxas de cicatrização.

\section{Fileira simples $\mathbf{x}$ fileira dupla}

Apreleva et al. ${ }^{39}$ mostraram que o reparo do manguito rotador com âncoras em fileira única (FU) consegue restaurar apenas $67 \%$ do footprint normal, o que poderia ser uma explicação para as falhas de cicatrização. Diante disso, Lo e Burkhart ${ }^{40}$ criaram a técnica de fileira dupla (FD) de âncoras. Essa técnica consiste em utilização de uma fileira de âncoras medial, junto à margem articular, e outra fileira no aspecto lateral do footprint. Dessa maneira, a largura de medial para lateral é reestabelecida, na tentativa de recriar a inserção do manguito rotador e, assim, otimizar o potencial de cicatrização. Do ponto de vista biomecânico, a adição de uma segunda fileira de âncoras aumenta o número de pontos de fixação, aumentando a força inicial da construção, diminuindo a carga que cada nó/âncora precisa resistir e diminuindo o stress no ponto de contato entre cada sutura no manguito. Estudos biomecânicos deram suporte à técnica, demonstrando diminuição significativa na formação de intervalo "gap" e deformação "strain," associado a aumento da força e rigidez inicial, se comparados à técnica de fileira simples. $^{41}$

Apesar de demonstrada vantagem biomecânica, o valor clínico da técnica é controverso, com parte importante dos ensaios afirmando não haver melhoras nos escores de qualidade de vida ou clínicos, apesar de vantagem na taxa de cicatrização da FD sobre a FU. ${ }^{42}$ Um estudo recente brasileiro, com resultados de 1 a 4 anos da técnica de fileira dupla corrobora com este fato, demonstrando não haver diferença estatisticamente significante nos escores UCLA e ASES (do inglês, American Shoulder and Elbow Score) entre as duas técnicas. ${ }^{43}$

Dado o exposto, para se tomar decisão sobre a real vantagem da técnica, e qual paciente se beneficiaria da mesma, Xu et al. $^{44}$ realizaram uma meta-análise, com ensaios clínicos randomizados de níveis 1 e 2 de evidência, comparando os dois métodos. Chegaram à conclusão que, apesar da técnica de FD apresentar menor taxa de nova ruptura (23,8\% FD x 40,2\% FU), melhor ASES e melhora na amplitude de rotação medial, não há diferença no escore de Constant, UCLA escore, elevação anterior, rotação lateral e força muscular se comparado à técnica de fileira única na análise geral dos resultados. Entretanto, ao fazer análise de subgrupos por tamanho de lesão, nas lesões grandes $>3 \mathrm{~cm}$, o reparo em fileira dupla mostrou melhora estatisticamente significante na cicatrização, UCLA e ASES. Esse resultado é corroborado por outros autores, como o sumário de metaanálises realizados por Spiegl et al. ${ }^{45}$ e a meta-análise produzida apenas com ensaios nível 1 de evidência, realizado por Sheibani-Rad et al. ${ }^{46}$

\section{Artroscópico $\mathrm{x}$ aberto}

Os métodos mais utilizados para a reconstrução do manguito rotador são o aberto, mini-open e artroscópico. Tanto a técnica mini-open quanto a artroscópica mantêm a integridade da origem do deltoide. Em contrapartida, na técnica aberta, parte do deltoide é desinserida da borda do acrômio. Teoricamente, essa seria a principal desvantagem da reconstrução aberta; contudo, o estudo de Cho et al., ${ }^{47}$ em análise de ressonância magnética (RM) pós-operatória, mostrou não haver diferença significativa entre as técnicas aberta e artroscópica no que diz respeito a complicações como destacamento da origem do deltoide ou alterações do músculo. Outra desvantagem da técnica aberta seria o aumento da dor na fase inicial pós-operatória, já que exige maior mobilização de partes moles. Este fato também não se confirmou, como demonstrado em meta-análise com estudos controle randomizados nível 1 realizado por Ji et al. ${ }^{48}$ 
Com relação aos índices de cicatrização, uma meta-análise recente, com estudos de alto nível de qualidade e 770 pacientes, mostrou taxas de cicatrização semelhante entre as técnicas mini-open e artroscópica. ${ }^{49}$ Ademais, não houve diferença significativa nos escores clínicos entre as técnicas.

Apesar da semelhança entre os resultados clínicos e taxas de cicatrização, vale lembrar que a técnica artroscópica oferece vantagem por proporcionar fácil acesso à articulação glenoumeral, sendo assim associada a um melhor resultado cosmético.

\section{Fatores pós-operatórios}

\section{Fatores químicos: anti-inflamatórios}

A cicatrização é um processo gradual, composto por fases sobrepostas, sendo a fase inflamatória mandatória. Qualquer fator que atrase ou altere o progresso da mesma pode afetála. Grande parte dos estudos sobre o assunto são in vitro ou com modelos animais, e, como constatado por Constantinescu et al., ${ }^{50}$ em revisão sistemática, a literatura atual não fornece evidência suficiente a favor ou contra o uso dos antiinflamatórios não esteroides (AINEs). Vale destacar que essa revisão encontrou apenas um ensaio clínico randomizado nível 1 de evidência. $O$ estudo em questão, realizado por Oh et al., ${ }^{51}$ comparou os efeitos analgésicos e efeitos colaterais no uso de celecoxibe (inibidor seletivo COX-2), ibuprofeno (AINE não seletivo) e tramadol (opioide). Nesses quesitos, não foram encontradas diferenças significativas entre as medicações. Em um segundo tempo, de modo retrospectivo, foram avaliadas, por RM e ultrassonografia (US), as taxas de cicatrização, tendo sido observado efeito negativo importante com o uso do inibidor seletivo de COX-2 (nova ruptura: $37 \%$ celecoxibe $\times 7 \%$ ibuprofeno $\times 4 \%$ tramadol). Por isso, apesar de apresentarem analgesia semelhante no pós-operatório, se comparados com outros AINEs e opioides, os inibidores seletivos da COX-2 não devem ser usados, já que podem atuar negativamente na cicatrização pós-reparo do manguito rotador.

\section{Fatores mecânicos}

A forma de se conduzir o pós-operatório pode interferir no processo de cicatrização do manguito rotador após uma reconstrução cirúrgica. Os protocolos de reabilitação levam em consideração a necessidade de proteção inicial da reconstrução, associada ao intuito de restaurar a função do ombro, prevenindo rigidez articular e atrofia da musculatura. A rigidez articular do ombro tem etiologia ainda obscura, porém pode estar relacionada à imobilização prolongada ou a programas de reabilitação conservadores. ${ }^{52}$ Estudos biomecânicos mostram que a carga precoce é prejudicial à organização das fibras de colágeno, podendo gerar microdano na interface osso/tendão, prevenindo assim a integração das fibras colágeno ao osso ou até a falha completa da cicatrização. ${ }^{53}$

\section{Mobilização precoce $\mathrm{x}$ mobilização tardia}

Os protocolos podem ser divididos basicamente em duas categorias: mobilização passiva precoce (early passive motion [EPM]) e imobilização com movimentação passiva tardia (immobilization with delayed range motion [DRM]). ${ }^{54}$ O protocolo EPM consiste em imobilização mínima com tipoia, em que são permitidos movimentos pendulares acrescidos de movimentos passivos de amplitude de movimento, como rotação lateral e elevação, desde o primeiro dia de pósoperatório. Já o protocolo DRM impõe utilização de tipoia sem realização de movimentos passivos, sendo permitidos apenas exercícios pendulares entre 2 a 4 semanas de pósoperatório. Realizando análise e revisão de meta análises publicadas, Houck et al..$^{55}$ chegaram à conclusão que o protocolo EPM melhora a amplitude de movimento, mas aumenta o risco de nova ruptura do manguito. Vale salientar que, apesar da rigidez do ombro ser um problema importante do pós-operatório da cirurgia do manguito rotador, a mesma é considerada uma complicação, diferente da recorrência da ruptura, que é considerada uma falha do tratamento.

\section{Tempo de utilização da tipoia}

Uma das primeiras perguntas dos pacientes na consulta préoperatória diz respeito ao tempo de utilização da tipoia. Existe bastante divergência na literatura com relação ao tempo mínimo de utilização associada à melhor cicatrização do manguito rotador. Koh et al. ${ }^{56}$ mostraram, em estudo nível 1 de evidência, que não há benefícios na cicatrização ao se comparar imobilização por 4 semanas e imobilização por 8 semanas. Portanto, a literatura não dá suporte à utilização de tipoia por tempo superior a 4 semanas.

\section{Tipoia Velpeau x tipoia de abdução}

Basicamente, a tipoia anti rotacional mantém o braço junto ao corpo em rotação medial. Já a tipoia de abdução mantém o ombro em variadas configurações de abdução e rotação lateral. Na análise biomecânica, Jackson et al. ${ }^{57}$ encontraram a posição ideal para criar o menor nível de tensão do supraespinhal e infraespinhal. Essa posição seria com elevação de 21 a $45^{\circ}$ e rotação lateral de 18 a $23^{\circ}$. No entanto, na prática, os resultados não comprovam os benefícios dessa posição. Alguns autores mostram que não houve diferença significativa em aspectos clínicos ou na taxa de nova ruptura. Outros estudos favoráveis à utilização de tipoia de abdução mostram vantagens do ponto de vista clínico da dor e função, sem avaliação dos resultados nas taxas de cicatrização. ${ }^{58}$ Vale ressaltar que, no geral, os estudos sobre imobilização com tipoia são feitos tendo como base as respostas do autoavaliação dos pacientes em relação à quantidade de tempo de uso da tipoia; porém, um questionamento importante é se esses pacientes realmente utilizam a tipoia como reportado. Tendo esse questionamento como base, Grubhofer et al. ${ }^{59}$ mostraram, avaliando utilização de tipoia de abdução com sensores digitais, que apenas $48 \%$ dos pacientes faziam o uso da tipoia de um modo considerado por eles como satisfatório ( $>80 \%$ do tempo). Além disso, ao comparar os dados obtidos pelos sensores e as informações dos pacientes, a discrepância foi assustadora, principalmente nos pacientes que não utilizavam a tipoia como recomendado. Os autores, portanto, sugerem cautela com os resultados de estudos sobre tipo e tempo de utilização das tipoias. 


\section{Fatores genéticos}

Figueiredo et al. ${ }^{60}$ identificaram fatores genéticos associados à susceptibilidade da lesão do manguito rotador, reforçando o papel da homeostase da matriz extracelular nesse contexto.

\section{Considerações finais}

Esse artigo de revisão possibilitou ampliarmos o entendimento acerca dos fatores que interferem no processo de cicatrização dos tendões do manguito rotador. Ficou patente que são muitas as variáveis, sendo elas locais, sistêmicas, mecânicas, químicas, genéticas ou associadas a hábitos de vida. Fatores pré, per e pós-operatórios influenciam de maneira decisiva nesse processo. Como mensagem final, sugere-se que o tratamento das lesões do manguito rotador seja feito de forma individualizada, levando-se em consideração todos os fatores descritos previamente e, nos pacientes idosos, antes da opção cirúrgica, que a tentativa do tratamento conservador deve ser considerada.

\section{Conflito de interesses}

Os autores declaram não haver conflito de interesses.

\section{Referências}

1 Yamanaka K, Fukuda H, Hamada K, Nakajima T. Histology of the supraspinatus tendon with reference to rotator cuff tears. In: Gazielly DF, Gleyze P, Thomas T, editors. The Cuff. New York: Elsevier; 1997:15-18

2 Uthoff HK, Ishii H. Histology of the cuff and pathogenesis of the degenerative tendinopathies. In: Gazielly DF, Gleyze P, Thomas T, editors. The Cuff. New York: Elsevier; 1997:19-23

3 Yamaguchi K, Tetro AM, Blam O, Evanoff BA, Teefey SA, Middleton WD. Natural history of asymptomatic rotator cuff tears: a longitudinal analysis of asymptomatic tears detected sonographically. J Shoulder Elbow Surg 2001;10(03):199-203

4 Milgrom C, Schaffler M, Gilbert S, van Holsbeeck M. Rotator-cuff changes in asymptomatic adults. The effect of age, hand dominance and gender. J Bone Joint Surg Br 1995;77(02):296-298

5 Carvalho AL, Martinelli F, Tramujas L, Baggio M, Crocetta MS, Martins RO. Lesões do manguito rotador e fatores associados à reoperação. Rev Bras Ortop 2016;51(03):298-302

6 Tashjian RZ, Hung M, Burks RT, Greis PE. Influence of preoperative musculotendinous junction position on rotator cuff healing using single-row technique. Arthroscopy 2013;29(11):1748-1754

7 Boileau P, Brassart N, Watkinson DJ, Carles M, Hatzidakis AM, Krishnan SG. Arthroscopic repair of full-thickness tears of the supraspinatus: does the tendon really heal? J Bone Joint Surg Am 2005;87(06):1229-1240

8 Galatz LM, Ball CM, Teefey SA, Middleton WD, Yamaguchi K. The outcome and repair integrity of completely arthroscopically repaired large and massive rotator cuff tears. J Bone Joint Surg Am 2004;86(02):219-224

9 Ikemoto RY, Murachovsky J, Nascimento LG, et al. Arthroscopic Repair Of Small And Medium Tears of The Supraspinatus Muscle Tendon: Evaluation of The Clinical And Functional Outcomes After Two Years Of Follow-Up. Rev Bras Ortop 2012;47(04):436-440

10 Godinho GG, França FO, Freitas JM, et al. Evaluation of anatomical integrity using ultrasound examination, and functional integrity using the constant \& murley score, of the rotator cuff following arthroscopic repair. Rev Bras Ortop 2010;45(02):174-180

11 Wylie JD, Baran S, Granger EK, Tashjian RZ. A Comprehensive Evaluation of Factors Affecting Healing, Range of Motion,
Strength, and Patient-Reported Outcomes After Arthroscopic Rotator Cuff Repair. Orthop J Sports Med 2018;6(01): 2325967117750104

12 Mochizuki T, Sugaya H, Uomizu M, et al. Humeral insertion of the supraspinatus and infraspinatus. New anatomical findings regarding the footprint of the rotator cuff. J Bone Joint Surg Am 2008;90 (05):962-969

13 Burkhart SS, Esch JC, Jolson RS. The rotator crescent and rotator cable: an anatomic description of the shoulder's "suspension bridge". Arthroscopy 1993;9(06):611-616

14 Meyer DC, Farshad M, Amacker NA, Gerber C, Wieser K. Quantitative analysis of muscle and tendon retraction in chronic rotator cuff tears. Am J Sports Med 2012;40(03):606-610

15 Jeong HY, Kim HJ, Jeon YS, Rhee YG. Factors Predictive of Healing in Large Rotator Cuff Tears: Is It Possible to Predict Retear Preoperatively? Am J Sports Med 2018;46(07):1693-1700

16 Khair MM, Lehman J, Tsouris N, Gulotta LV. A Systematic Review of Preoperative Fatty Infiltration and Rotator Cuff Outcomes. HSS J 2016;12(02):170-176

17 Sethi PM, Sheth CD, Pauzenberger L, et al. Macroscopic Rotator Cuff Tendinopathy and Histopathology Do Not Predict Repair Outcomes of Rotator Cuff Tears. Am J Sports Med 2018;46(04): 779-785

18 Clark JM, Harryman DT 2nd. Tendons, ligaments, and capsule of the rotator cuff. Gross and microscopic anatomy. J Bone Joint Surg Am 1992;74(05):713-725

19 Boileau P, Andreani O, Schramm M, Baba M, Barret H, Chelli M. The Effect of Tendon Delamination on Rotator Cuff Healing. Am J Sports Med 2019;47(05):1074-1081

20 Kwon J, Lee YH, Kim SH, Ko JH, Park BK, Oh JH. Delamination Does Not Affect Outcomes After Arthroscopic Rotator Cuff Repair as Compared With Nondelaminated Rotator Cuff Tears: A Study of 1043 Consecutive Cases. Am J Sports Med 2019;47(03):674-681

21 Heuberer PR, Plachel F, Willinger L, et al. Critical shoulder angle combined with age predict five shoulder pathologies: a retrospective analysis of 1000 cases. BMC Musculoskelet Disord 2017; 18(01):1-9

22 Moor BK, Bouaicha S, Rothenfluh DA, Sukthankar A, Gerber C. Is there an association between the individual anatomy of the scapula and the development of rotator cuff tears or osteoarthritis of the glenohumeral joint?: A radiological study of the critical shoulder angle Bone Joint J 2013;95-B(07):935-941

23 Gomide LC, Carmo TC, Bergo GH, Oliveira GA, Macedo IS. Associação entre o ângulo crítico do ombro e lesão do manguito rotador: um estudo epidemiológico retrospectivo. Rev Bras Ortop 2017;52(04):423-427

24 Gerber C, Snedeker JG, Baumgartner D, Viehöfer AF. Supraspinatus tendon load during abduction is dependent on the size of the critical shoulder angle: A biomechanical analysis. J Orthop Res 2014;32(07):952-957

25 Garcia GH, Liu JN, Degen RM, et al. Higher critical shoulder angle increases the risk of retear after rotator cuff repair. J Shoulder Elbow Surg 2017;26(02):241-245

26 Scheiderer B, Imhoff FB, Johnson JD, et al. Higher Critical Shoulder Angle and Acromion Index Are Associated With Increased Retear Risk After Isolated Supraspinatus Tendon Repair at Short-Term Follow Up. Arthroscopy 2018;34(10):2748-2754

27 Passaretti D, Candela V, Venditto T, Giannicola G, Gumina S. Association between alcohol consumption and rotator cuff tear. Acta Orthop 2016;87(02):165-168

28 Park JH, Oh KS, Kim TM, et al. Effect of Smoking on Healing Failure After Rotator Cuff Repair. Am J Sports Med 2018;46(12): 2960-2968

29 Cho NS, Moon SC, Jeon JW, Rhee YG. The influence of diabetes mellitus on clinical and structural outcomes after arthroscopic rotator cuff repair. Am J Sports Med 2015;43(04):991-997

30 Gumina S, Arceri V, Carbone S, et al. The association between arterial hypertension and rotator cuff tear: the influence on 
rotator cuff tear sizes. J Shoulder Elbow Surg 2013;22(02): 229-232

31 Beason DP, Hsu JE, Marshall SM, et al. Hypercholesterolemia increases supraspinatus tendon stiffness and elastic modulus across multiple species. J Shoulder Elbow Surg 2013;22(05): 681-686

32 Gumina S, Candela V, Passaretti D, et al. The association between body fat and rotator cuff tear: the influence on rotator cuff tear sizes. J Shoulder Elbow Surg 2014;23(11):1669-1674

33 Thankam FG, Dilisio MF, Agrawal DK. Immunobiological factors aggravating the fatty infiltration on tendons and muscles in rotator cuff lesions. Mol Cell Biochem 2016;417(1-2):17-33

34 Chung SW, Kim JY, Kim MH, Kim SH, Oh JH. Arthroscopic repair of massive rotator cuff tears: outcome and analysis of factors associated with healing failure or poor postoperative function. Am J Sports Med 2013;41(07):1674-1683

35 Cho NS, Rhee YG. The factors affecting the clinical outcome and integrity of arthroscopically repaired rotator cuff tears of the shoulder. Clin Orthop Surg 2009;1(02):96-104

36 Veado MA, Prata EF, Gomes DC. Lesão do manguito rotador em pacientes maiores de 65 anos: avaliação da função, integridade e força. Rev Bras Ortop 2015;50(03):318-323

37 Dezaly C, Sirveaux F, Philippe R, et al. Arthroscopic treatment of rotator cuff tear in the over-60s: repair is preferable to isolated acromioplasty-tenotomy in the short term. Orthop Traumatol Surg Res 2011;97(6, Suppl)S125-S130

38 Mall NA, Tanaka MJ, Choi LS, Paletta GA Jr. Factors affecting rotator cuff healing. J Bone Joint Surg Am 2014;96(09):778-788

39 Apreleva M, Ozbaydar M, Fitzgibbons PG, Warner JJ. Rotator cuff tears: the effect of the reconstruction method on three-dimensional repair site area. Arthroscopy 2002;18(05):519-526

40 Lo IK, Burkhart SS. Double-row arthroscopic rotator cuff repair: re-establishing the footprint of the rotator cuff. Arthroscopy 2003;19(09):1035-1042

41 Smith CD, Alexander S, Hill AM, et al. A biomechanical comparison of single and double-row fixation in arthroscopic rotator cuff repair. J Bone Joint Surg Am 2006;88(11):2425-2431

42 Charousset C, Grimberg J, Duranthon LD, Bellaiche L, Petrover D. Can a double-row anchorage technique improve tendon healing in arthroscopic rotator cuff repair?: A prospective, nonrandomized, comparative study of double-row and single-row anchorage techniques with computed tomographic arthrography tendon healing assessment Am J Sports Med 2007;35(08):1247-1253

43 Senna LF, Ramos MR, Bergamaschi RF. Reparo artroscópico do manguito rotador: fileira simples versus fileira dupla - Resultados clínicos após um a quatro anos. Rev Bras Ortop 2018;53(04): $448-453$

$44 \mathrm{Xu} \mathrm{C}$, Zhao J, Li D. Meta-analysis comparing single-row and double-row repair techniques in the arthroscopic treatment of rotator cuff tears. J Shoulder Elbow Surg 2014;23(02):182-188

45 Spiegl UJ, Euler SA, Millett PJ, Hepp P. Summary of Meta-Analyses Dealing with Single-Row versus Double-Row Repair Techniques for Rotator Cuff Tears. Open Orthop J 2016;10:330-338
46 Sheibani-Rad S, Giveans MR, Arnoczky SP, Bedi A. Arthroscopic single-row versus double-row rotator cuff repair: a meta-analysis of the randomized clinical trials. Arthroscopy 2013;29(02): 343-348

47 Cho NS, Cha SW, Rhee YG. Alterations of the Deltoid Muscle After Open Versus Arthroscopic Rotator Cuff Repair. Am J Sports Med 2015;43(12):2927-2934

48 Ji X, Bi C, Wang F, Wang Q. Arthroscopic versus mini-open rotator cuff repair: an up-to-date meta-analysis of randomized controlled trials. Arthroscopy 2015;31(01):118-124

49 Shan L, Fu D, Chen K, Cai Z, Li G. All-arthroscopic versus mini-open repair of small to large sized rotator cuff tears: a meta-analysis of clinical outcomes. PLoS One 2014;9(04):e94421

50 Constantinescu DS, Campbell MP, Moatshe G, Vap AR. Effects of Perioperative Nonsteroidal Anti-inflammatory Drug Administration on Soft Tissue Healing: A Systematic Review of Clinical Outcomes After Sports Medicine Orthopaedic Surgery Procedures. Orthop J Sports Med 2019;7(04):2325967119838873

51 Oh JH, Seo HJ, Lee YH, Choi HY, Joung HY, Kim SH. Do Selective COX-2 Inhibitors Affect Pain Control and Healing After Arthroscopic Rotator Cuff Repair? A Preliminary Study. Am J Sports Med 2018;46(03):679-686

52 Koo SS, Burkhart SS. Rehabilitation following arthroscopic rotator cuff repair. Clin Sports Med 2010;29(02):203-211

53 Gerber C, Schneeberger AG, Perren SM, Nyffeler RW. Experimental rotator cuff repair. A preliminary study. J Bone Joint Surg Am 1999;81(09):1281-1290

54 Bakti N, Antonios T, Phadke A, Singh B. Early versus delayed mobilization following rotator cuff repair. J Clin Orthop Trauma 2019;10(02):257-260

55 Houck DA, Kraeutler MJ, Schuette HB, McCarty EC, Bravman JT. Early Versus Delayed Motion After Rotator Cuff Repair: A Systematic Review of Overlapping Meta-analyses. Am J Sports Med 2017;45(12):2911-2915

56 Koh KH, Lim TK, Shon MS, Park YE, Lee SW, Yoo JC. Effect of immobilization without passive exercise after rotator cuff repair: randomized clinical trial comparing four and eight weeks of immobilization. J Bone Joint Surg Am 2014;96(06):e44

57 Jackson M, Sylvestre É, Bleau J, Allard P, Begon M. Estimating optimal shoulder immobilization postures following surgical repair of massive rotator cuff tears. J Biomech 2013;46(01):179-182

58 Conti M, Garofalo R, Castagna A. Does a brace influence clinical outcomes after arthroscopic rotator cuff repair? Musculoskelet Surg 2015;99(Suppl 1):S31-S35

59 Grubhofer F, Gerber C, Meyer DC, et al. Compliance with wearing an abduction brace after arthroscopic rotator cuff repair: A prospective, sensor-controlled study. Prosthet Orthot Int 2019; 43(04):440-446

60 Figueiredo EA, Loyola LC, Belangero PS, et al. Rotator Cuff Tear Susceptibility Is Associated With Variants in Genes Involved in Tendon Extracellular Matrix Homeostasis. J Orthop Res 2020;38 (01):192-201 